\title{
Impact of COVID-19 on interest in hepato-pancreato-biliary diseases
}

\author{
Haig Pakhchanian ${ }^{1}$ (D) $\cdot$ Rahul Raiker ${ }^{2} \cdot$ Sinan Kardeş $^{3} \cdot$ Mohammad Bilal $^{4} \cdot$ Khushnuma Alam $^{1} \cdot$ Ahmad Khan $^{2} \cdot$ \\ William Hutson $^{2}$. Shyam Thakkar ${ }^{2}$. Shailendra Singh ${ }^{2}$
}

Received: 17 June 2021 / Accepted: 16 August 2021 / Published online: 23 August 2021

(C) The Author(s), under exclusive licence to Springer-Verlag GmbH Germany, part of Springer Nature 2021

\begin{abstract}
An important gap of knowledge exists regarding the public interest in hepato-pancreato-biliary (HPB) diseases during the coronavirus disease 2019 (COVID-19) pandemic. We aimed to understand the public interest in HPB diseases in the COVID19 era. In this infodemiology study, we performed a comparative analysis of Google search volume of HPB terms in 2020-2021 and compared it to a similar time frame (2016-2019) in 3 periods to assess how trends in patient seeking behavior of HPB terms changed during the course of the pandemic in the USA and worldwide. Our analysis showed a substantial decrease in search volume of HPB diseases and procedure terms early in the pandemic. However, search volumes appeared to revert back to prepandemic years closer to the 1-year mark in USA and worldwide. Patients may have initially neglected HPB-related issues during the early phase of the COVID-19 pandemic, which could lead to worse outcomes. While HPB-related terms reverted closer to pre-pandemic levels later in the pandemic, further research is needed to assess the long-term impacts.
\end{abstract}

Keywords Infodemiology $\cdot$ Health services $\cdot$ Liver $\cdot$ Gallbladder $\cdot$ Pancreas $\cdot$ COVID-19

\section{Introduction}

Google Trends has been widely used in assessing temporal and geographical public interest in various topics through assessing relative Google search volume (Eysenbach 2006). In addition, Google Trends has been leveraged to generate epidemic models for real-time prediction of coronavirus

Haig Pakhchanian and Rahul Raiker contributed equally to this work

Responsible Editor: Lotfi Aleya

Haig Pakhchanian

haig@gwmail.gwu.edu

Shailendra Singh

Shail121@gmail.com

Rahul Raiker

rsraiker@gmail.com

Sinan Kardeș

sinan.kardes@istanbul.edu.tr

Mohammad Bilal

mbilal@bidmc.harvard.edu

Khushnuma Alam

alamkhoshay@gmail.com

Ahmad Khan

drahmadkhan83@gmail.com disease 2019 (COVID-19) (Peng et al. 2021) and investigate public interest in diverse medical fields including musculoskeletal diseases (Kardeș et al. 2021), dermatology (Kutlu 2020), mental health (Becerra-García et al. 2021), and pediatric neurosurgery (Güdük et al. 2021) during the COVID-19 pandemic. All these previous studies indicate Google Trends being a valuable tool for evaluation of public interest.

William Hutson

whuston@hsc.wvu.edu

Shyam Thakkar

sthakkar@hsc.wvu.edu

Department of Gastroenterology, George Washington University School of Medicine \& Health Science, Washington, DC, USA

2 Department of Digestive Diseases, West Virginia University School of Medicine, Morgantown, WV, USA

3 Department of Medical Ecology and Hydroclimatology, Istanbul Faculty of Medicine, Istanbul University, Istanbul, Turkey

4 Department of Gastroenterology, University of Minnesota/ Minneapolis VA Medical Center, Minneapolis, MN, USA 
Disruption of healthcare services during early phase of the COVID-19 pandemic could have led to the neglect of nonCOVID-19 medical problems, including hepato-pancreatobiliary (HPB)-related diseases and procedures. Moreover, the increase in alcohol consumption and an unhealthy lifestyle during the pandemic (Pollard et al. 2020; Bennett et al. 2021; Tison et al. 2020) may worsen HPB diseases. However, information is currently limited as how patient search interest varied throughout the 1-year period of the pandemic.

Therefore, the aim of this study was to analyze trends in Google searches to understand the public interest in HPB diseases during the different stages of the COVID-19 pandemic through a comparative analysis of the search volume of commonly used HPB terms compared to previous years.

\section{Materials and methods}

We assessed Google search volume for commonly used HPB terms individually and organized them into four categories: liver disease-related terms, liver test- or procedure-related terms, pancreato-biliary disease-related terms, and pancreato-biliary-related test or procedure-related terms. For each search term, relative search volume (RSV) was collected which is a value that is normalized on a scale from 0 to 100 . An RSV value of 100 represents the highest search volume over a selected time, while a value of 0 represents the least amount of search volume. Google Trends provides average weekly RSV values which was used for analysis.

Search terms were assessed for RSV using filters for region, date, and category. Two search strategies were utilized. One assessed RSV using the parameters "United States," "Jan 1, 2016, to Feb 28th, 2021," and "all categories." The second search strategy assessed global RSV using the parameters "Worldwide," "Jan 1, 2016, to Feb 28th, 2021," and "All Categories."

\section{Statistical analyses}

Weekly RSV data from January 1, 2020, to February 28, 2021, for USA and worldwide was compared to the combined mean weekly RSV data of the corresponding location for a similar time (2016-2019) in three periods of the COVID-19 pandemic: March 1-June 28, July 5-November 1, and November 8-February 28. A $t$-test of two independent samples assuming unequal variances was utilized to compare the two groups in public interest during the different stages of the COVID-19 pandemic. Comparisons with a two-sided $p$-value $<0.05$ was considered statistically significant. All analyses were conducted on Microsoft Excel 2019 (Microsoft Corporation, Redmond, Washington). The presentation of statistical results was in line with recent review (Misra et al. 2021).

\section{Results}

A total of six liver disease, five liver test or procedure, seven pancreato-biliary disease, and six pancreato-biliary test- or procedure-related terms were included for analysis. The results are detailed in Table 1.

In the first period of the pandemic, the USA and worldwide both saw statistically significant decreases in searches when compared to searches during previous years. These terms included the following: cirrhosis, hepatitis, liver cancer, varices, liver biopsy, liver function test, liver transplant, MELD score, gallbladder cancer, gallstones, pancreatic cancer, pancreatitis, endoscopic retrograde cholangiopancreatography (ERCP), HIDA scan, magnetic resonance cholangiopancreatography (MRCP), and gallbladder surgery. There was a significant decrease in biliary stricture searches but significant increase in jaundice and endoscopic ultrasonography (EUS) searches in worldwide searches alone. Lastly, there was a significant decrease in searches for biliary colic and pancreas surgery in the USA alone. The remaining 3 terms showed no differences from prior years in both the USA and worldwide.

During the second period of the pandemic, USA and worldwide searches both saw a statistically significant decrease in hepatitis, pancreatic cancer, and HIDA scan but significant increases in cirrhosis and non-alcoholic fatty liver disease (NAFLD) from prior years. There was a significant increase in searches for paracentesis, cholangitis, pancreatitis, and EUS worldwide whereas in USA alone, there was a significant decrease in searches for liver cancer, and gallbladder cancer. The other 12 terms showed no differences from prior years in both USA and worldwide.

During the third period of the pandemic, US and worldwide searches showed significant decrease in hepatitis, liver cancer, and HIDA scan. In addition, there was a significant decrease in searches for biliary stricture and gallstones in the USA alone. Worldwide searches showed a significant decrease in searches for liver function tests, and liver transplant but a significant increase in cirrhosis, pancreatitis, and EUS. The other 13 terms showed no differences in search volume from prior years in both USA and worldwide.

\section{Discussion}

Our findings suggest that the overall public interest in HPBrelated diseases and procedures in the USA and worldwide decreased during the first phase of the COVID-19 pandemic but then rebounded back to previous years' levels of interest from a similar pre-pandemic timeframe during the second and third periods. This is evident by the initial reduction of the relative search volume of 15 of 24 HPB search terms followed by a reduction in searches for only three HPB-related search terms during the second and third periods of the pandemic. 


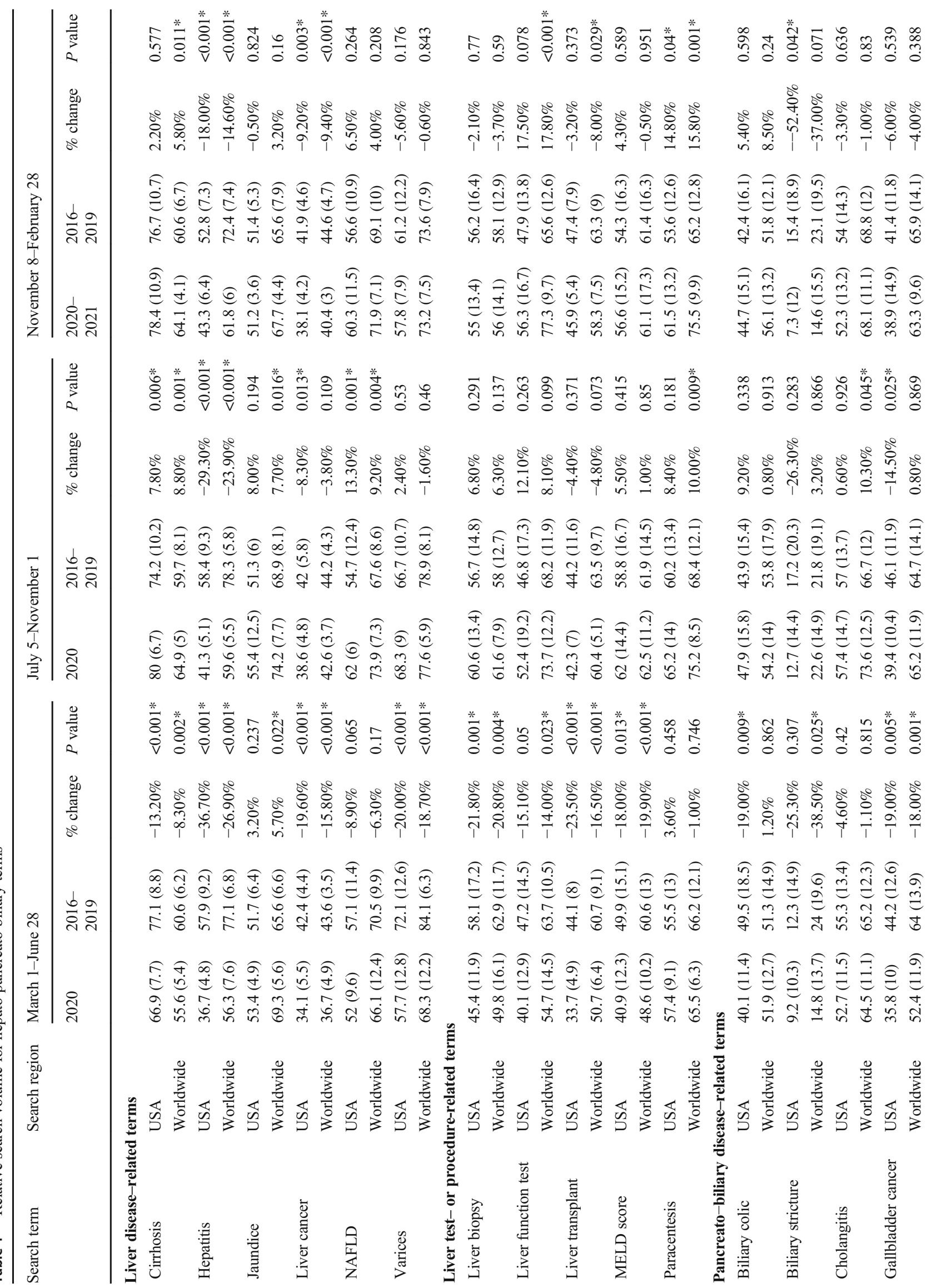




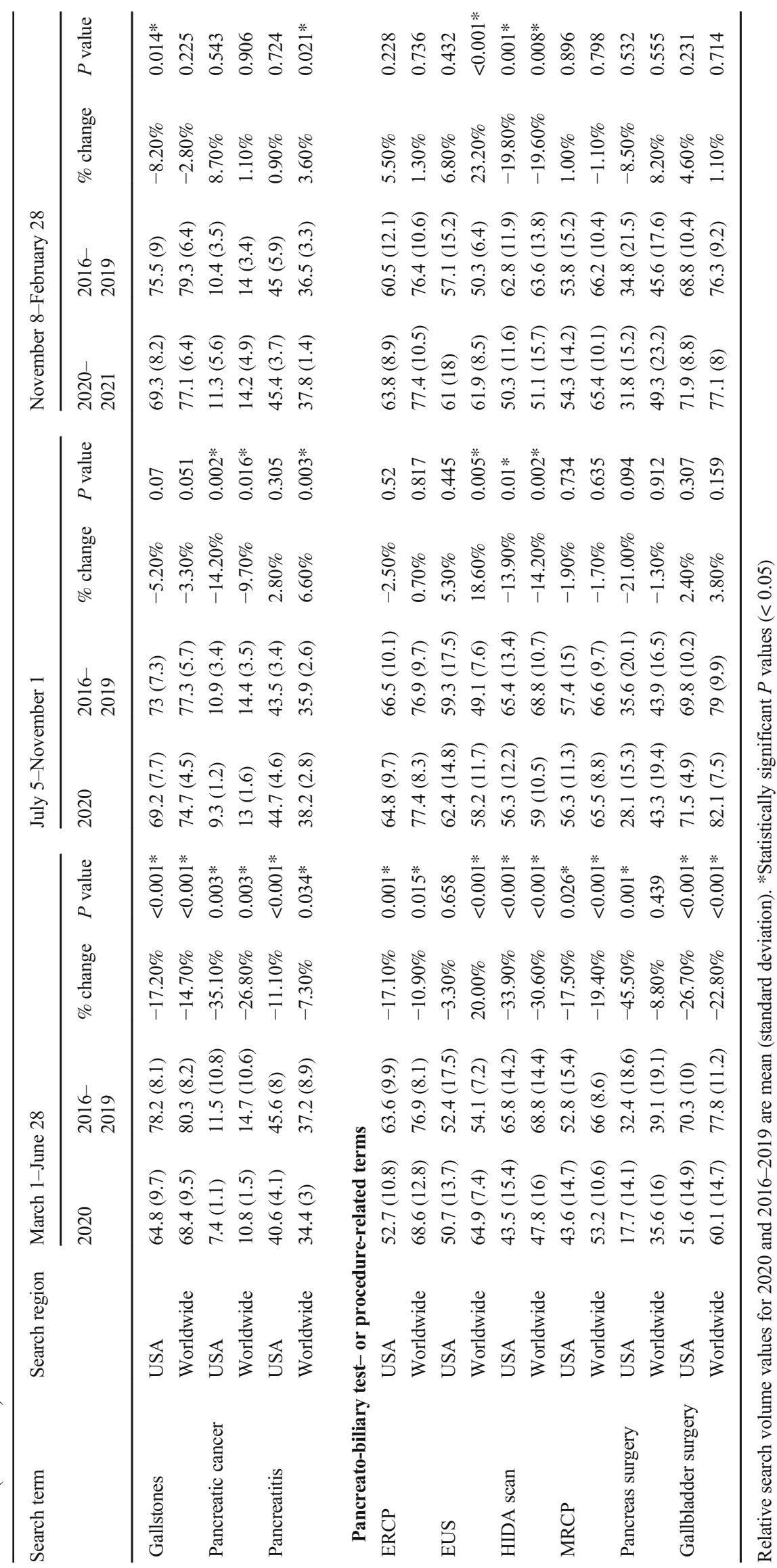


During the first period, public interest was mostly focused on COVID-19, which likely led to a decline in attention for nonCOVID-19-related health issues. Another factor could have been a significant decrease in patient presentation to healthcare centers due to fear of exposure to severe acute respiratory syndrome coronavirus 2 (SARS-CoV-2) (Anderson et al. 2020); hence, there was a decrease in patients receiving a new diagnosis of HPB-related conditions when compared to previous years. Similarly, many of the HPBrelated procedures were deferred early in the COVID-19 pandemic to a later period in the pandemic when more concrete guidelines and safety measures were set in place (Sawhney et al. 2020).

While there was a reduction in most of the search terms related to HBP conditions, there was an increase in the searches related to the term "jaundice" and "EUS." While the COVID-19-associated liver injury leading to jaundice (Paliogiannis and Zinellu 2020) might contribute to the initial observed rise in jaundice searches in our study, the exact reason for this is unclear.

Previous reports evaluating Google Trends also showed similar trends in relative search volume among common diseases and procedures in gastroenterology, along with other fields of medicine, e.g., otolaryngology and dermatology (Pier et al. 2020; Guzman and Barbieri 2020). In a previous study, Rajan et al. (2020) investigated the Google searches of 4 common gastrointestinal symptoms associated with COVID-19 (i.e., diarrhea, nausea, vomiting, and abdominal pain). They showed that the relative search volume of nausea, vomiting, and abdominal pain remained near or below baseline; however, an increase was seen in searches for diarrhea in early March 2020. Our study, as the second gastrointestinalCOVID-19 study via Google Trends, provides insight into public interest in hepato-pancreato-biliary-related search terms in the COVID-19 era. However, future studies investigating public interest in other gastrointestinal diseases during the COVID-19 pandemic through Google Trends analysis are needed.

Similarly, Esen-Salman et al. (2021) utilized Google Trends to show an initial decrease in US RSV of dermatology symptoms, conditions, treatments, and procedural search terms followed by a revert to pre-pandemic RSV levels. One hundred twenty search terms were queried during the first (March 15-May 9), second (May 10-July 4), and third (July 5-October 31) periods of the pandemic compared to overlapping periods during the preceding 4 years, respectively. Fiftytwo, 28, and 19 search terms showed a statistically significant decrease in RSV during each respective period, while 18, 36, and 36 search terms showed increases, respectively. These results coincide with Google search trends among HPBrelated terms as majority of each queried term showed an initial decrease with a subsequent increase to pre-pandemic RSV levels.
The initial reduction in interest in HPB-related medical conditions and procedures among the general public during the COVID-19 pandemic could have potential implications. Patients may have neglected HPB-related issues and symptoms given a shifted focus to COVID-19. This could lead to a future surge in HPB-related diseases and procedures even after the pandemic ends. Many of the HPB-related cancers such as cholangiocarcinoma, pancreas, and gall bladder cancer already present at a later stage in the disease course. Neglecting or overlooking symptoms associated with these diseases can further worse outcomes. These factors, coupled with previously mentioned points such as a tendency towards an unhealthy lifestyle during the pandemic and increased alcohol consumption (Arora and Grey 2020), can also worsen pre-existing HPB disorders such as chronic liver disease and diseases of the pancreas. Gastroenterologists should be prepared to meet the possible backlog of HPB-related diseases and procedures as restrictions slowly are uplifted and patients feel more comfortable to seek healthcare as vaccination becomes more accessible. Moreover, as COVID-19 begins to dwindle, it is essential to continue educating patients regarding various HPB diseases by using local resources and other social and electronic media platforms.

\section{Limitations}

Our study has several limitations. The search volume data does not allow to establish direct causation. While our study shows that there has been a decrease in Google search interest in HBP-related conditions and procedures with a subsequent rise in later periods, there is no evidence to suggest this could have an impact on outcomes. Second, we only analyzed one search engine. However, the Google search engine has approximately $90 \%$ of the market share (Statcounter GlobalStats. 2021); it is likely representative of all Internet searches.

\section{Conclusion}

Our study is the first study to portray a 1-year analysis of trends in public interest of HPB-related terms during the COVID pandemic, which shows that there has been a decrease in the public interest for the majority of HPB-related conditions and procedures and a rebound to usual public interest that is similar to previous pre-pandemic time frames. Further studies are needed to evaluate the implications of Google search trends on actual patient behaviors and conducting modeling studies to predict the impact on disease burden and outcomes. 
Author contribution H. P., R. R., and S. S. contributed to data curation, formal analysis, and original manuscript drafting. H. P., R. R., S. K., M. B., K. A., A. K., W. H., S. T., and S. S. contributed to conceptualization, investigation, methodology, and manuscript review and editing. H. P., R. R., S. K., M. B., K. A., A. K., W. H., S. T., and S. S. approved the final draft submitted.

Data availability Data are available from corresponding authors upon a reasonable request.

\section{Declarations}

Ethics approval and consent to participate Not applicable.

Consent for publication Not applicable.

Competing interests The authors declare no competing interests.

\section{References}

Anderson TS, Stevens JP, Pinheiro A, Li S, Herzig SJ (2020) Hospitalizations for emergent medical, surgical, and obstetric conditions in boston during the COVID-19 pandemic. J Gen Intern Med 35:3129-3132. https://doi.org/10.1007/s11606-020-06027-2

Arora T, Grey I (2020) Health behaviour changes during COVID-19 and the potential consequences: a mini-review. J Health Psychol 25: 1155-1163. https://doi.org/10.1177/1359105320937053

Becerra-García JA, Sánchez-Gutiérrez T, Barbeito S, Calvo A (2021) COVID-19 pandemic and mental health in Spain: an analysis of their relationship using Google Trends. Rev Psiquiatr Salud Ment. https://doi.org/10.1016/j.rpsm.2021.05.001

Bennett G, Young E, Butler I, Coe S (2021) The impact of lockdown during the COVID-19 outbreak on dietary habits in various population groups: a scoping review. Front Nutr 8:626432. https://doi.org/ $10.3389 /$ fnut.2021.626432

Esen-Salman K, Akın-Çakıcı Ö, Kardeș S, Salman A (2021) Public interest in dermatologic symptoms, conditions, treatments, and procedures during the COVID-19 pandemic: Insights from Google Trends. Dermatol Ther 34:e14895. https://doi.org/10.1111/dth. 14895

Eysenbach G (2006) Infodemiology: tracking flu-related searches on the web for syndromic surveillance. AMIA Annu Symp Proc 2006: 244-248

Güdük M, Orhun Ö, Dursun AT, Küçüksüleymanoğlu D, Deniz Z, Usseli MI, Bozkurt B, Kardeș S, Ekși MȘ (2021) Impact of COVID-19 on interest in pediatric neurosurgery related symptoms, diseases, and treatments. J Neurosurg Sci. https://doi.org/10.23736/S0390-5616. 21.05416-3
Guzman AK, Barbieri JS (2020) Analysis of dermatology-related search engine trends during the COVID-19 pandemic: implications for patient demand for outpatient services and telehealth. J Am Acad Dermatol 83:963-965. https://doi.org/10.1016/j.jaad.2020.05.147

Kardeș S, Erdem A, Gürdal H (2021) Public interest in musculoskeletal symptoms and disorders during the COVID-19 pandemic: infodemiology study. Z Rheumatol. https://doi.org/10.1007/ s00393-021-00989-2

Kutlu Ö (2020) Analysis of dermatologic conditions in Turkey and Italy by using Google Trends analysis in the era of the COVID-19 pandemic. Dermatol Ther 33:e13949. https://doi.org/10.1111/dth. 13949

Misra DP, Zimba O, Gasparyan AY (2021) Statistical data presentation: a primer for rheumatology researchers. Rheumatol Int 41:43-55. https://doi.org/10.1007/s00296-020-04740-z

Paliogiannis P, Zinellu A (2020) Bilirubin levels in patients with mild and severe Covid-19: a pooled analysis. Liver Int 40:1787-1788. https:// doi.org/10.1111/liv.14477

Peng Y, Li C, Rong Y, Pang CP, Chen X, Chen H (2021) Real-time prediction of the daily incidence of COVID-19 in 215 countries and territories using machine learning: model development and validation. J Med Internet Res 23:e24285. https://doi.org/10.2196/ 24285

Pier MM, Pasick LJ, Benito DA, Alnouri G, Sataloff RT (2020) Otolaryngology-related Google Search trends during the COVID19 pandemic. Am J Otolaryngol 41:102615. https://doi.org/10. 1016/j.amjoto.2020.102615

Pollard MS, Tucker JS, Green HD Jr (2020) Changes in adult alcohol use and consequences during the COVID-19 pandemic in the US. JAMA Netw Open 3:e2022942. https://doi.org/10.1001/ jamanetworkopen.2020.22942

Rajan A, Sharaf R, Brown RS, Sharaiha RZ, Lebwohl B, Mahadev S (2020) Association of search query interest in gastrointestinal symptoms with COVID-19 diagnosis in the United States: infodemiology study. JMIR Public Health Surveill 6:e19354. https://doi.org/10. 2196/19354

Sawhney MS, Bilal M, Pohl H, Kushnir VM, Khashab MA, Schulman AR, Berzin TM, Chahal P, Muthusamy VR, Varadarajulu S, Banerjee S, Ginsberg GG, Raju GS, Feuerstein JD (2020) Triaging advanced GI endoscopy procedures during the COVID-19 pandemic: consensus recommendations using the Delphi method. Gastrointest Endosc 92:535-542. https://doi.org/10.1016/j.gie. 2020.05 .014

Statcounter GlobalStats. 2021. https://gs.statcounter.com/search-enginemarket-share/.

Tison GH, Avram R, Kuhar P, Abreau S, Marcus GM, Pletcher MJ, Olgin JE (2020) Worldwide effect of COVID-19 on physical activity: a descriptive study. Ann Intern Med 173:767-770. https://doi.org/10. 7326/M20-2665

Publisher's note Springer Nature remains neutral with regard to jurisdictional claims in published maps and institutional affiliations. 